necessarily long proofs of theorems), are usually due to one of two phenomena: First, there is a true redundancy, whereby two phases of the computation (proof) merely cancel each other out. See [6] for the analysis of such a theorem proof. Secondly, the computation (proof) does more than is really needed, or wanted. See [7] for such a theorem proof. In our present case the inefficiency is clearly of the second type.

Finally, we note that D. H. Lehmer, in [4], showed how most of the subtractions in (10) could also be eliminated. It is not necessary to compute every $\gamma_{n} / \gamma_{n+1}$, but merely those at periodic intervals. It was his method that was used here in computing Table 1. Any interested reader will now have no difficulty in determining precisely how Lehmer manages to obtain this still greater efficiency.

David Taylor Model Basin

Washington, D. C. 20007

1. Daniel Shanks, (Reviewer), RMT 11, 12, (Review of two translations of Khintchine's Continued Fractions), Math. Comp., v. 20, 1966, pp. 171-173.

2. Daniel Shanks \& J. W. Wrench, JR., "Khintchine's constant," Amer. Math. Monthly, v. 66,1959 , pp. 276-279. MR $21 \# 1950$.

3. J. W. WRENCH, JR., "Further evaluation of Khintchine's constant," Math. Comp., v. 14, 1960 , pp. $370-371$.

4. D. H. Lehmer, "Euclid's Algorithm for large numbers," Amer. Math. Monthly, v. 45, 1938, pp. 227-233.

5. D. E. KNUth, "Euler's constant to 1271 places," Math. Comp., v. 16, 1962, pp. 275-281. MR $26 \# 5763$.

6. DANiel Shanks, "A study of postulates: The 'thermodynamic' derivation of the adiabatic gas law," Amer. J. Phys., v. 24, 1956, pp. 352-354.

7. Daniel Shanks, "Is the quadratic reciprocity law a deep theorem?" Solved and Unsolved Problems in Number Theory, Vol. 1, Spartan, Washington, 1962, p. 65, see (a). MR 28 $\# 3952$.

\title{
Distributions of Mersenne Divisors
}

\section{By Sidney Kravitz}

By driving computers to the limit of their capability, 23 prime Mersenne Numbers have been discovered [1]. The list of known divisors on the other hand is a large one. As a result of both of these lists, conjectures have appeared regarding the expected number of primes and of divisors [1], [2], [3]. This note presents additional data relative to the observed frequency of divisors of Mersenne Numbers.

Each divisor, $q$, of the Mersenne Number $M_{p}=2^{p}-1, p$ a prime, is of the form $2 k p+1$ and of the form $8 L \pm 1$. (Therefore $k \neq 4 n+2$.) Thus if $k$ is known for a particular $p$, it identifies the divisor. The divisors of the Mersenne Numbers, $3 \leqq p$ $<100,000$ have been examined for $k \leqq 200$. The frequency $f$, with which the various values of $k$ occur is given in Table 1. This table shows that the frequency of $k$ tends to decrease as $k$ increases, but those $k$ with a large number of small divisors, e.g. 12, 24, and 60, occur with much greater frequency than their neighbors on the list.

Received November 29, 1965. 
TABLE 1

\begin{tabular}{|c|c|c|c|c|c|c|c|c|c|c|c|c|c|}
\hline$k$ & $f$ & $k$ & $f$ & $k$ & $f$ & $k$ & $f$ & $k$ & $f$ & $k$ & $f$ & $k$ & $f$ \\
\hline 1 & 581 & 33 & 32 & 65 & 7 & 97 & 7 & 129 & 12 & 161 & 5 & 193 & 2 \\
\hline 3 & 350 & 35 & 13 & 67 & 8 & 99 & 3 & 131 & 6 & 163 & 5 & 195 & 6 \\
\hline 4 & 266 & 36 & 41 & 68 & 12 & 100 & 12 & 132 & 11 & 164 & 5 & 196 & 6 \\
\hline 5 & 141 & 37 & 17 & 69 & 10 & 101 & 4 & 133 & 2 & 165 & 7 & 197 & 1 \\
\hline 7 & 84 & 39 & 29 & 71 & 8 & 103 & 4 & 135 & 6 & 167 & 3 & 199 & 0 \\
\hline 8 & 122 & 40 & 25 & 72 & 28 & 104 & 9 & 136 & 4 & 168 & 10 & 200 & 7 \\
\hline 9 & 115 & 41 & 11 & 73 & 5 & 105 & 10 & 137 & 5 & 169 & 3 & & \\
\hline 11 & 61 & 43 & 10 & 75 & 10 & 107 & 3 & 139 & 2 & 171 & 10 & & \\
\hline 12 & 162 & 44 & 19 & 76 & 10 & 108 & 17 & 140 & 6 & 172 & 3 & & \\
\hline 13 & 47 & 45 & 24 & 77 & 7 & 109 & 4 & 141 & 9 & 173 & 2 & & \\
\hline 15 & 88 & 47 & 11 & 79 & 6 & 111 & 8 & 143 & 1 & 175 & 2 & & \\
\hline 16 & 56 & 48 & 39 & 80 & 20 & 112 & 10 & 144 & 15 & 176 & 7 & & \\
\hline 17 & 23 & 49 & 6 & 81 & 11 & 113 & 8 & 145 & 4 & 177 & 6 & & \\
\hline 19 & 26 & 51 & 16 & 83 & 6 & 115 & 6 & 147 & 3 & 179 & 1 & & \\
\hline 20 & 56 & 52 & 15 & 84 & 27 & 116 & 9 & 148 & 5 & 180 & 7 & & \\
\hline 21 & 47 & 53 & 8 & 85 & 5 & 117 & 6 & 149 & 2 & 181 & 2 & & \\
\hline 23 & 18 & 55 & 14 & 87 & 9 & 119 & 5 & 151 & 5 & 183 & 6 & & \\
\hline 24 & 68 & 56 & 30 & 88 & 16 & 120 & 13 & 152 & 5 & 184 & 4 & & \\
\hline 25 & 34 & 57 & 15 & 89 & 8 & 121 & 4 & 153 & 7 & 185 & 1 & & \\
\hline 27 & 27 & 59 & 7 & 91 & 5 & 123 & 9 & $15 \tilde{5}$ & 4 & 187 & 2 & & \\
\hline 28 & 37 & 60 & 47 & 92 & 12 & 124 & 7 & 156 & 13 & 188 & 1 & & \\
\hline 29 & 11 & 61 & 5 & 93 & 6 & 125 & 6 & 157 & 2 & 189 & 8 & & \\
\hline 31 & 16 & 63 & 17 & 95 & 5 & 127 & 2 & 159 & 2 & 191 & 1 & & \\
\hline 32 & 24 & 64 & 17 & 96 & 16 & 128 & 8 & 160 & 5 & 192 & 13 & & \\
\hline
\end{tabular}

This writer advocates that the $k$ values for each divisior be listed in future tables of divisors rather than the divisor itself, inasmuch as the $k$ reveals more about the character of the divisor [4], [5], [6], [7], [8].

The referee points out that well-known heuristic arguments, cf. [3, Example $39 \mathrm{~S}$, p. 214], would suggest that $f(1)$ is asymptotic, but rather slowly, and from below, to one-half the number of twin primes up to the same limit. Further, cf. [3, Example 16, pp. 29, 169], one expects that $f(3)$ is asymptotic, again slowly from below, to $\frac{2}{3}$ of $f(1)$. Comparison of the data here with the 1224 twins up to 100,000 is satisfactory.

\section{Dover, New Jersey}

1. D. B. Gillies, "Three new Mersenne primes and a statistical theory," Math. Comp., v. 18,1964 , pp. $93-97$. MR $28 \# 2990$.

2. I. J. Good, "Conjectures concerning the Mersenne Numbers," MTAC, v. 9, 1955, pp. 120-121. MR 17, 127.

3. D. Shanks, Solved and Cnsolved Problems in Number Theory, Vol. I, Spartan, Washington, D. C., 1962, p. 198. MR $28 \# 3952$.

4. S. KRAVitz \& J. S. MADACHY, "Divisors of Mersenne Numbers, 20,000<p<100,000," deposited in the UMT File, reviewed by D. Shanks, Math. Comp., v. 19, 1965, p. 686.

5. E. Karst, "Search limits on divisors of Mersenne Numbers," Nordisk Tidskr. Informations-Behandlung, v. 2, 1962, pp. 224-227. MR $29 \# 3422$.

6. E. KARST, "Some new divisors of Mersenne Numbers," BIT 2, 1962, p. 90.

7. S. KRAvitz, "Divisors of Mersenne Numbers $10,000<p<15,000$," Math. Comp., v. 15, 1961 , pp. $292-293$. MR $23 \# 833$.

8. V. A. Golubev, "Nombres de Mersenne et caractères du nombre 2," Mathesis, v. 67, 1958 , pp. $257-262$. MR $21 \# 28$. 